Title Page

\title{
Applying and Optimizing Water-Soluble, Slow-Release Nitrogen Fertilizers for Water-Saving Agriculture
}

Yanle Guo ${ }^{\mathrm{a}, \mathrm{b}}$, Min Zhang ${ }^{\mathrm{a},{ }^{,}}$, Zhiguang Liu ${ }^{\mathrm{a},{ }^{*}}$, Chenhao Zhao ${ }^{\mathrm{a}}$, Hao Lu ${ }^{\mathrm{a}}$, Lei Zheng ${ }^{\mathrm{c}}$,

Yuncong C. $\mathrm{Li}^{\mathrm{d}}$

${ }^{a}$ National Engineering Laboratory for Efficient Utilization of Soil and Fertilizer Resources, National Engineering \& Technology Research Center for Slow and Controlled Release Fertilizers, College of Resources and Environment, Shandong Agricultural University, Tai'an, Shandong 271018, China

${ }^{\mathrm{b}}$ College of Horticulture and Landscape Architecture, Jinling Institute of Technology, Nanjing 210038, China

'State Key Laboratory of Nutrition Resources Integrated Utilization, Kingenta Ecological Engineering Group Co., Ltd, Linshu, Shandong 276700, China ${ }^{\mathrm{d}}$ Department of Soil and Water Science, Tropical Research and Education Center, IFAS, University of Florida, Homestead, FL 33031, USA

* Corresponding authors: Tel.: +86-538-8241531; fax: +86-538-8241531

E-mail addresses: minzhang-2002@163.com (M. Zhang);

liuzhiguang8235126@126.com (Z. Liu). 
Table S1. The single-factor test design

\begin{tabular}{ccccc}
\hline Run & $\begin{array}{c}\mathrm{U}^{\mathrm{a}}{ }^{\mathrm{a}} \\
\text { (molar ratio) }\end{array}$ & $\begin{array}{c}\mathrm{F} \mathrm{NH}_{3}{ }^{\mathrm{b}} \\
\text { (molar ratio) }\end{array}$ & $\begin{array}{c}\text { Reaction } \\
\text { temperature }\left({ }^{\circ} \mathrm{C}\right)\end{array}$ & $\begin{array}{c}\text { Reaction time } \\
(\mathrm{h})\end{array}$ \\
\hline 1 & 1.75 & 1.90 & 60 & 2.5 \\
2 & 1.50 & 2.22 & 60 & 2.5 \\
3 & 1.25 & 2.67 & 60 & 2.5 \\
4 & 1.00 & 3.33 & 60 & 2.5 \\
5 & 0.75 & 4.44 & 60 & 2.5 \\
\hline 6 & 1.25 & 4.00 & 60 & 2.5 \\
7 & 1.25 & 3.33 & 60 & 2.5 \\
8 & 1.25 & 2.67 & 60 & 2.5 \\
9 & 1.25 & 2.00 & 60 & 2.5 \\
10 & 1.25 & 1.00 & 60 & 2.5 \\
\hline 11 & 1.25 & 2.67 & 20 & 2.5 \\
12 & 1.25 & 2.67 & 40 & 2.5 \\
13 & 1.25 & 2.67 & 60 & 2.5 \\
14 & 1.25 & 2.67 & 80 & 2.5 \\
15 & 1.25 & 2.67 & 100 & 2.5 \\
\hline 16 & 1.25 & 2.67 & 60 & 0.5 \\
17 & 1.25 & 2.67 & 60 & 1.5 \\
18 & 1.25 & 2.67 & 60 & 2.5 \\
19 & 1.25 & 2.67 & 60 & 3.5 \\
20 & 1.25 & 2.67 & 60 & 4.5 \\
\hline
\end{tabular}

${ }^{a}$ Urea:formaldehyde ratio.

${ }^{\mathrm{b}}$ Formaldehyde:amine ratio. 


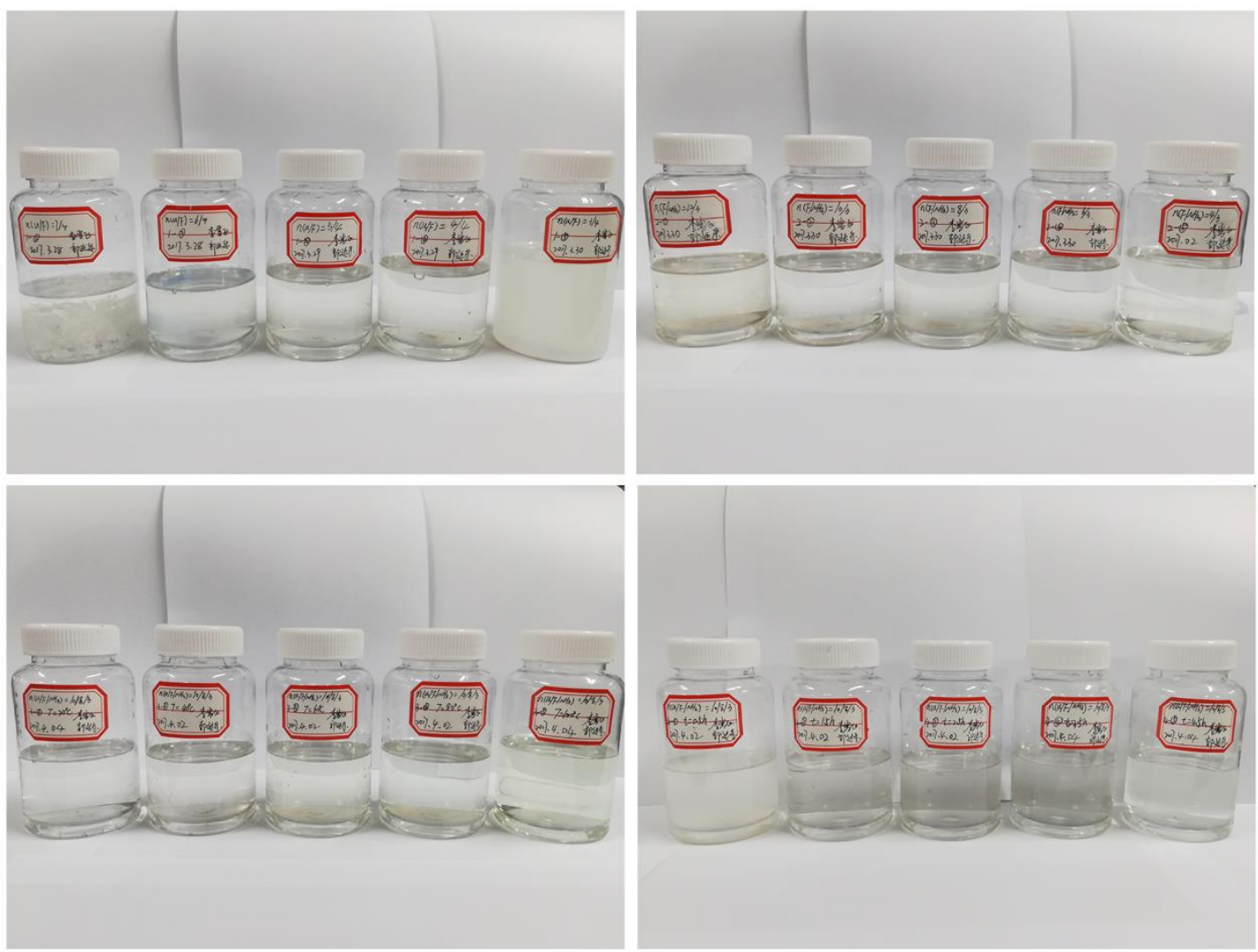

Figure S1. Samples of single-factor experiments 

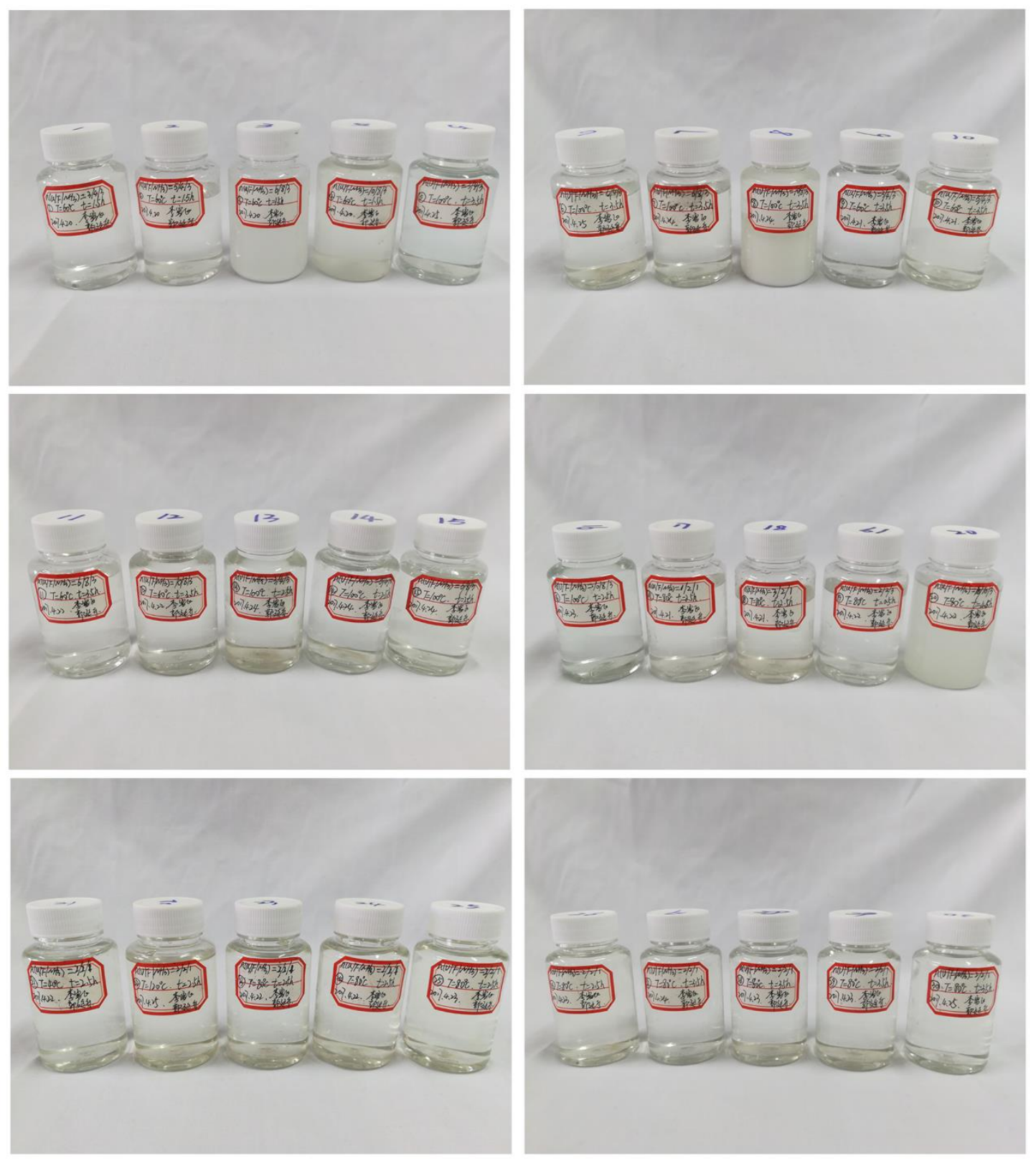

Figure S2. Samples of response surface methodology design 